\title{
Resección quirúrgica de plasmocitoma solitario del esternón asociado a cirugía de revascularización miocárdica. Seguimiento a largo plazo*
}

\author{
Drs. ROBERTO GONZÁLEZ L. ${ }^{1}$, RODRIGO CAMPOS M. ${ }^{1}$, \\ ENRIQUE SEGUEL S. ${ }^{1}$, ALECK STOCKINS L. ${ }^{1}$, HERIBERTO FARÍAS M. ${ }^{1}$, \\ LUIS NEIRA S. ${ }^{1}$, RENÉ SALDÍAS F. ${ }^{1}$, EMILIO ALARCÓN C. ${ }^{1}$ \\ 1 Equipo de Cirugía Cardiotorácica, Hospital "Dr. Guillermo Grant Benavente”. Facultad de Medicina, \\ Universidad de Concepción. \\ Concepción, Chile.
}

\begin{abstract}
Surgical resection of solitary plasmacytoma of the sternum associated with coronary artery bypass grafting surgery. Long-term follow up
\end{abstract}

The plasmacytomas tumors are caused by a malignant proliferation of plasma cells. Primary tumors of the sternum are uncommon, being this kind of tumors exceptional. We present the case of a 72 years old man with a sternal plasmacytoma, that after 2 years of receiving radiotherapy treatment, had tumor growth, associated with coronary artery disease. In this case, surgical resection of solitary sternal plasmacytoma was performed associated with coronary artery bypass grafting surgery. The chest wall reconstruction was done with a polypropylene mesh. More than 5 years long-term follow up was completed and the patient is asymptomatic and free of malignant proliferation of plasma cells.

Key words: Plasmacytoma, sternum, radiotherapy, coronary artery disease, coronary artery bypass.

\section{Resumen}

Los plasmocitomas son tumores originados por una proliferación neoplásica de células plasmáticas. Los tumores primarios de esternón son infrecuentes, siendo los plasmocitomas solitarios de esternón excepcionales. Presentamos el caso de un hombre de 72 años con un plasmocitoma esternal que después de 2 años post tratamiento con radioterapia presentó aumento de tamaño tumoral asociado a enfermedad coronaria. Se realizó resección quirúrgica del plasmocitoma solitario del esternón asociada a cirugía de revascularización miocárdica con 4 bypass coronarios. La pared torácica fue reconstituida con una malla de polipropileno. A más de 5 años de seguimiento el paciente se encuentra asintomático cardiovascular y libre proliferación maligna de células plasmáticas.

Palabras clave: Plasmocitoma, esternón, radioterapia, enfermedad coronaria, revascularización miocárdica.

*Recibido el 24 de julio de 2010 y aceptado para publicación el 19 de octubre de 2010.

No existen conflictos de interés ni apoyo financiero.

Correspondencia: Dr. Roberto González L.

Janequeo esquina Chacabuco, Concepción, Chile. Fax: 56-41-2204881

rgonzalezlagos@udec.cl 


\section{Introducción}

Los plasmocitomas son tumores originados por una proliferación neoplásica de células plasmáticas, pueden estar más o menos delimitados y se presentan en ausencia de mieloma múltiple. Cuando estos tumores afectan al tejido óseo, se denominan plasmocitoma solitario de hueso y cuando afectan a tejidos blandos, plasmocitoma extramedular ${ }^{1}$.

Los tumores primarios de esternón son infrecuentes, siendo los plasmocitomas solitarios de esternón excepcionales ${ }^{2-5}$.

No encontramos comunicaciones de casos en que se presente un plasmocitoma solitario de esternón asociado a enfermedad coronaria y en que se realizara tratamiento quirúrgico simultáneo. Presentamos un caso de resección quirúrgica de un plasmocitoma solitario del esternón asociado a cirugía de revascularización miocárdica con seguimiento a largo plazo.

\section{Caso clínico}

Hombre de 72 años sin antecedentes de importancia, presentó aumento de volumen paraesternal derecho indoloro por lo que consultó en otro centro. Se realizó una biopsia quirúrgica de la lesión y el estudio de anatomía patológica concluyó que la lesión era una neoplasia de células plasmáticas tipo plasmocitoma. Con estos antecedentes fue derivado al equipo de hematología de nuestro hospital. Se completó el estudio con cintigrama óseo, electroforesis de proteínas, inmunoglobulinas séricas, $\beta-2$ microglubulina y mielograma. Se descartó mieloma múltiple diagnosticándose plasmocitoma solitario del esternón y se realizó radioterapia recibiendo un total de $70 \mathrm{~Gy}$.

El paciente se mantuvo con el aumento de volumen esternal indoloro y en controles periódicos con hematólogo y radioterapeuta sin evidenciar progresión de la enfermedad o mieloma múltiple.

A dos años de finalizada la radioterapia, el paciente evolucionó con dolor retroesternal tipo anginoso y el estudio con cintigrama miocárdico con mibi-dipiridamol demostró necrosis antero apical e isquemia postero inferior. La coronariografía demostró lesiones coronarias significativas proximales de tres vasos con buenos lechos dístales y una función ventricular conservada con akinesia apical. Se mantuvo con tratamiento médico para la cardiopatía coronaria sin respuesta y con progresión de la angina. En esta etapa de la evolución, el tumor presentó aumento de tamaño y dolor asociado, que el paciente diferenció claramente del dolor anginoso.

Se presentó a comité oncológico del hospital y a nuestro equipo de cirugía cardiotorácica, se decidió resección quirúrgica del plasmocitoma solitario del esternón asociado a cirugía de revascularización miocárdica. La tomografía computada de tórax previa a la cirugía, demostró un tumor en el cuerpo esternal de aproximadamente 15 centímetros con compromiso del pericardio y del plano muscular de la pared torácica anterior.

\section{Procedimiento quirúrgico}

Se realizó resección del tumor esternal, ésta incluyó: el cuerpo esternal, los cartílagos costales que se articulaban con el cuerpo esternal y tejidos blandos (incluidos fragmentos de pericardio y fragmentos de ambas pleuras que se encontraban infiltrados), se preservó el mango esternal que estaba libre de enfermedad. Terminada la cirugía resectiva del tumor, se conectó en circulación extracorpórea y se realizó cirugía de revascularización miocárdica. Se utilizaron sólo conductos venosos de safena interna, ya que ambas arterias mamarias internas estaban comprometidas en la masa tumoral. Los bypass realizados fueron: a la arteria coronaria descendente anterior, a una arteria diagonal, a una rama de la arteria coronaria circunfleja y a la rama descendente posterior de la arteria coronaria derecha. La salida de circulación extracorpórea fue sin incidentes.

El mango esternal se fijó con puntos de alambre de acero inoxidable y el defecto de la pared (Figura 1A) se reconstruyó con una malla de polipropileno. Los pectorales fueron suturados en la línea media cubriendo adecuadamente la malla.

\section{Evolución postoperatoria}

La evolución post operatoria fue sin incidentes siendo dado de alta hospitalaria al séptimo día post operatorio.

El estudio de anatomía patológica, informó una lesión tumoral de 15 centímetros y 680 gramos de peso (Figura 1B), que destruye el esternón y se extiende en profundidad a el pericardio y ambas pleuras. El tumor presentaba abundantes células plasmáticas mononucleares compatibles con el diagnóstico de plasmocitoma, el estudio con inmunohistoquímica confirmó con el diagnóstico. Los bordes quirúrgicos de resección estaban libres de enfermedad.

Desde el punto cardiovascular a los 5 años 2 meses post operado, presentó angina, la coronariografía demostró progresión de la enfermedad coronaria especialmente en la arteria circunfleja. Se realizó angioplastía con stent no medicado a la arteria circunfleja proximal. Los 4 bypass venosos se encontraban permeables con enfermedad no significativa.

El paciente a 5 años 6 meses de seguimiento, se ha mantenido sin evidencias clínicas, ni tomográficas de recidiva tumoral. En controles periódicos 


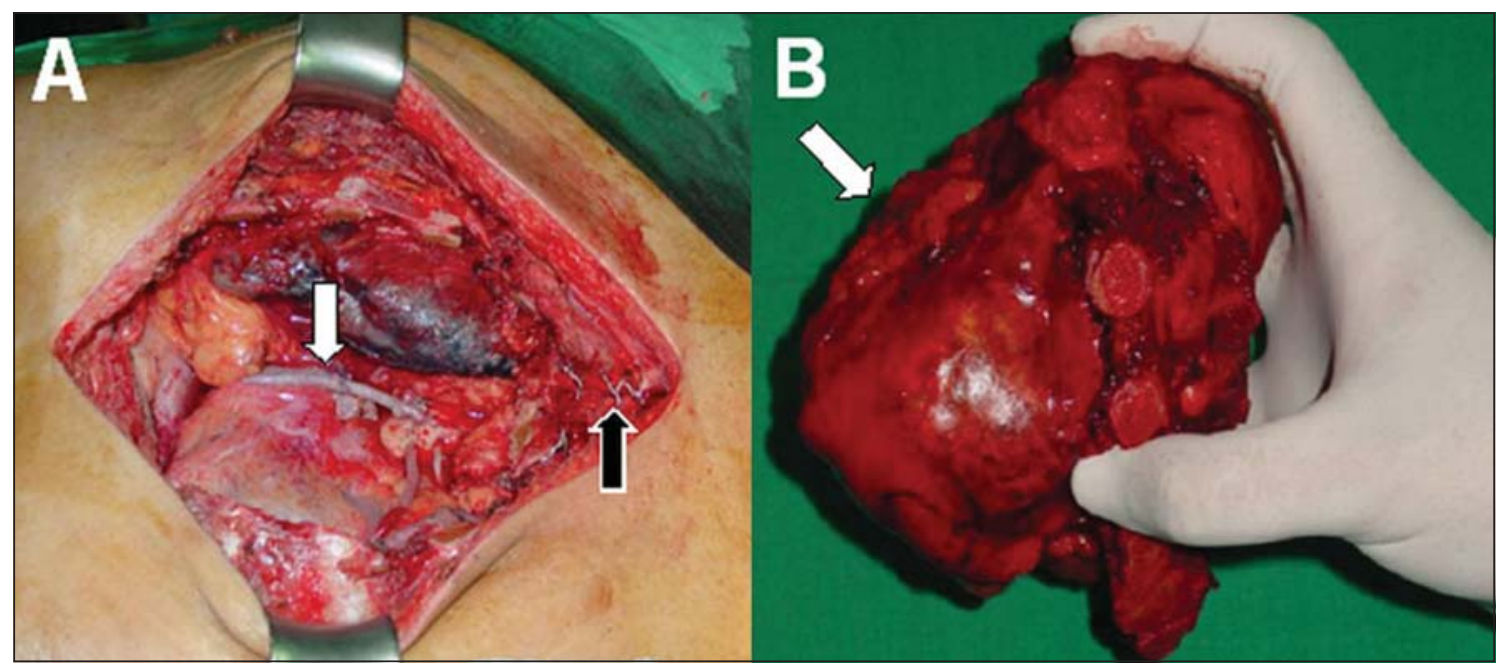

Figura 1. A. Defecto de pared torácica luego de resección del plasmocitoma, se observa mango esternal estabilizado con punto de alambre (flecha negra) y bypass coronario a la arteria coronaria derecha (flecha blanca). B. Se observa el mayor crecimiento tumoral intra torácico (flecha).

con hematólogo no ha evidenciado progresión de la enfermedad, ni mieloma múltiple. Actualmente se encuentra sin angina y realizando vida normal (Nivel 0, según escala Performance Status ECOG). Se realizó control con angio tomografía computada que no demostró evidencia de recidiva y los 4 bypass coronarios permeables.

\section{Discusión}

Las neoplasias de células plasmáticas son poco frecuentes, éstas pueden presentarse como: mieloma múltiple, plasmocitoma extramedular y plasmocitoma solitario de hueso. El mieloma múltiple es la forma de presentación más frecuente y traduce compromiso sistémico. Los plasmocitomas extramedulares se presentan como lesiones aisladas en tejidos blandos, son más frecuentes en la orofaringe y nasofaringe, al igual que los plasmocitomas solitarios de hueso, deben presentarse en ausencia de mieloma múltiple ${ }^{1}$.

Los plasmocitomas solitarios son tumores óseos infrecuentes y corresponden aproximadamente al $5 \%$ de los tumores de células plasmáticas. Se pueden localizar en cualquier hueso, son más frecuentes en la columna vertebral, fémur y cresta iliaca, en el tórax se han comunicado en las costillas y en el esternón ${ }^{1,4,6}$. Radiológicamente, los plasmocitomas solitarios de hueso se manifiestan como una lesión multiquística con destrucción de la porción medular del hueso ${ }^{7}$.

Los plasmocitomas solitarios se presentan en ausencia de mieloma múltiple, aunque, el $40 \%$ a $75 \%$ de los pacientes, desarrolla mieloma en el seguimiento. Incluso, se ha planteado que podrían corresponder a una etapa de la misma enfermedad ${ }^{1}$. Se manifiestan como aumento de volumen óseo, que puede ser indoloro según la velocidad de crecimiento, esta también es variable existiendo casos de crecimiento acelerado ${ }^{1,2,4}$. En el caso presentado, el tumor se mantuvo de similar tamaño por 2 años post radioterapia para luego presentar un crecimiento acelerado asociado a dolor.

Para asegurar que se trata de un plasmocitoma solitario se debe descartar la existencia de mieloma múltiple y en el seguimiento se debe tener presente que pueden asociase ${ }^{1}$. En este caso, existe un seguimiento mayor a 5 años libre de enfermedad. La supervivencia se relaciona con la aparición de mieloma múltiple y alcanza entre el $38 \%$ al $69 \%$ a 5 años ${ }^{4,5,8}$.

El plasmocitoma constituye un grupo excepcional entre los tumores esternales y su tratamiento no está del todo definido, la radioterapia y la cirugía son los tratamientos que han demostrado beneficios ${ }^{2-5,9}$. La resección quirúrgica con márgenes amplios y seguimiento adecuado son una alternativa válida de tratamiento ${ }^{2-5}$. Nuestro paciente, recibió radioterapia y luego de dos años el tumor continuó con aumento de tamaño, esto asociado a su cardiopatía coronaria llevó al equipo tratante a derivarlo al equipo de cirugía.

Conocidos son los efectos que la radioterapia produce sobre el corazón y en particular sobre las arterias coronarias ${ }^{10}$. En este caso en particular, la ausencia de factores de riesgo cardiovascular y la aparición de angina dos años post radioterapia, 
necesariamente hacen preguntarnos el rol que la radioterapia jugó y jugará en el desarrollo y evolución de la enfermedad coronaria.

La reconstrucción del defecto de la pared torácica luego de la resección tumoral se realizó con una malla de polipropileno, esto permitió una adecuada cobertura con resultados estéticos y funcionales satisfactorios. Un punto importante en nuestro caso, es que se pudo preservar el mango esternal, esto permitió una adecuada estabilidad de la pared torácica. La forma de reconstrucción de la pared torácica luego de resecciones tumorales debe ser evaluada en cada caso en particular, existiendo distintas alternativas para ello $11-15$.

No encontramos comunicaciones de pacientes con plasmocitoma solitario esternal y enfermedad coronaria que simultáneamente fueran tratados quirúrgicamente y evaluados con un seguimiento mayor de 5 años. En nuestro caso, destacamos el hecho que el paciente se encuentre libre de enfermedad neoplásica y asintomático cardiovascular con sus by pass venosos permeables y con una buena calidad de vida.

La resección quirúrgica con márgenes amplios y seguimiento adecuado son una alternativa válida de tratamiento en los plasmocitomas solitarios de esternón.

\section{Referencias}

1. Di Micco P, Di Micco B. Up-date on solitary plasmacytoma and its main differences with multiple myeloma. Exp Oncol. 2005;27:1-7.

2. Pezzella AT, Fall SM, Pauling FW, Sadler TR. Solitary plasmocytoma of the sternum: surgical resection with long-term follow-up. Ann Thorac Surg. 1989;48:859-62.

3. Athanassiadi K, Kalavrouziotis G, Rondogianni D, Loutsidis A, Hatzimichalis A, Bellenis I. Primary chest wall tumors: early and long-trem results of surgical treatmnet. Eur J Cardiothorac Surg. 2001;19:589-93.

4. Sabanathan S, Shah R, Merans AJ. Surgical treatment of primary malignat chest tumours. Eur J Cardiothorac Surg. 1997;11:1011-6.

5. Martini N, Huvos AG, Burt ME, Heelan RT, Bains MS, Mccormack PM, et al. Predictors of survival in malignant tumors of the sternum. J Thoracic Cardiovasc Surg. 1996;111:96-106.

6. García Franco CE, Jiménez Hiscock L, Zapatero Gaviria J. Plasmocitoma costal solitario. Arch Broncomeunol. 2004;40:100.

7. Tateishi U, Gladish GW, Kusumoto M, Hasegawa T, Yokoyama R, Tsuchiya R, et al. Chest wall tumors: radiologic findings an pathologic correlation. Radiographics 2003;23:1491-508.

8. Burt M, Karpeh M, Ukoha O, Bains MS, Martini N, Mccormack PM, et al. Medical tumors of the chest wall. Solitary plasmacytoma and Ewing's sarcoma. J Thorac Cardiovasc Surg. 1993;105:89-96.

9. Jyothirmay R, Gangadharan VP, Nair MK, Rajan B. Radiotherapy in the treatment of solitary plasmacytoma. The British Journal of Radiology 1997;70:511-6.

10. Heidenreich PA, Kapoor JR. Radiation induced heart disease: systemic disorders in heart disease. Heart 2009;95:252-8.

11. Puma F, Avenia N, Ricci F, Guiducci A, Fornasari V, Daddi G. Bone heterograft for chest wall reconstruction after sternal resection. Ann Thorac Surg. 1996;61:525-9.

12. Warzelhan J, Stoelben E, Imdahl A, Hasse J. Results in surgery for primary and metastatic chest wall tumors. Eur J Cardiothorac Surg. 2001;19:584-8.

13. Incarbone M, Pastorino U. Surgical treatment of chest wall tumors. World J Surg. 2001;25:218-30.

14. Manssur KA, Thourani VH, Losken A, Reeves JG, Millar JI, Carlson GW, et al. Chest wall resections and reconstruction: a 25-year experience. Ann Thorac Surg. 2002;73:1720-6.

15. Skoracki RJ, Chang DW. Reconstruction of the chest wall and thorax. J Surg Oncol. 2006;94:455-65. 